\title{
Blockchain in Robotic Distributed Multi-Level Systems
}

\author{
Alexey Beskopylny ${ }^{1 *}$, Aleksey Lysenko ${ }^{2}$ and Evgenii Garanin ${ }^{2}$ \\ ${ }^{1}$ Department Transport Systems and Traffic, Russia \\ ${ }^{2}$ Robotics resource centre, Russia
}

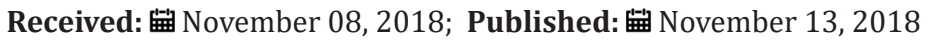

*Corresponding author: Alexey Beskopylny, Vice rector for education affairs and Advanced Degrees, Department Transport Systems and Traffic, Russia

\begin{abstract}
The problem of using Blockchain technology in multi-level robotic systems is considered. The management of the robotic systems faces significant difficulties in transferring large amounts of information, securities, metadata, and intellectual contracts. The blockchain technology, based on a decentralized system of distributed registries, allows solving data transfer problems quickly and safely. New digital blockchain-based queuing systems can be effectively used in multi-level control tasks.
\end{abstract}

Keywords: Blockchain; Decentralized; Robotic system

\section{Introduction}

In recent years, blockchain technologies have become widely used not only in financial markets and cryptocurrency systems but also in many others. Many practical Blockchain applications in intelligent transport systems, building information models in civil engineering, internet of things, energy systems and others make it possible to unveil unprecedented abilities to create and transfer valuable information. The survey presenting IoT security solutions published in [1] shows the potential for blockchain technology in facilitating secure sharing of IoT datasets and securing IoT systems. A new concept for a distributed space transportation system was proposed and a similar decentralized cooperative control scheme was investigated in [2]. A systematic control architecture that includes information flow of homogeneous space robots transporting a large object in orbit is developed. Largescale application of the Internet of Things technology requires improvement of security problems and formation of an integrated security system, for example, banking, logistics, insurance, contract law, and others. Some IoT environments where blockchain mechanisms play an essential role were considered in [3]. The useful application of blockchain technology in distributed decentralized energy systems (DES) is given in [4]. Blockchain allows DES to play an important role in energy services, such as meeting the business needs of energy producers and consumers, providing an entirely controlled and secure energy monitoring platform. The use of the Blockchain for secure, intelligent vehicle communication that can be used to track vehicle-generated data was proposed in [5]. A decentralized control algorithm for the robots to accomplish the comprehensive coverage was offered in [6].

The full coverage is achieved by coordinating the robots along a given path that is unknown to the vehicles. A control architecture that allows a multi-robot system to implement complex cooperative dynamic behaviors, defined as tracking of arbitrary periodic setpoints while avoiding collisions, was considered in [7]. A decentralized approach for conflict resolution where robots show reactive and safe behaviors, avoiding collisions with both static and dynamic objects was shown in [8]. The method was obtained for unicycle robots with range-finder sensors, and it was able to manage with noisy sensors and second order dynamic constraints. Thus, the large-scale application of blockchain technologies in decentralized multi-level systems allows reaching a new quality level of management.

\section{Problem Definition}

It is known that the centralized control system has many significant drawbacks in managing multi-level systems. Centralized solutions are not very well implemented in dynamic situations with a large number of robots: communication can cause internal system conflicts and may not be reliable enough; the complexity of algorithms grows with the number of robots, making the online processing of information too complicated in many situations. The position of the robot in space, interaction with other robots, 
the transmission of streaming information about the results of the work performed by the robot is dynamically changing and must be stored in the system, especially when the transfer of information is made in limited access mode. Thus, the application of various blockchain solutions and decentralized approach show great potential in different robotics-oriented tasks in logistics and automated sort facilities. The task of logistic systems is to control the flow of goods, their transportation, processing, and packaging. All logistics systems require mobility in either closed or open space. Automatically (or sometimes autonomously) controlled vehicles (AGVs) are mobile robots used for automatic material transfer from one point to another. The use of automated vehicles includes transportation and, depending on specific tasks, processing, packaging, sorting and delivery. The main advantage of robotic logistics solutions is a reduction in the need for manual labor, reduction in the human factor, safety in the workplace and greater accuracy in the inventory.

\section{Blockchain Enabled Robot Swarm}

Considering the abovementioned and the fact that one of the main obstacles to the large-scale deployment of robots for commercial applications is security it can be beneficial to use blockchain solutions for decentralized robotics control systems as shown in Figure 1. It is worth mentioning that robotic swarm systems have recently been gaining popularity [9] making it a highly applicable solution for controlling heterogeneous groups of robots due to their adaptability to different environments [10] and tasks [11] (Figure 1). The crucial role in the development of swarm systems plays the implementation of distributed decision-making algorithms. However, it is worth mentioning that in scenarios with a large number of robots with distributed decision-making remains an open problem [12]. The integration of blockchain technology in the robot swarms distributed decision-making algorithms can help develop more resilient and adaptive mission control for automated logistics tasks.

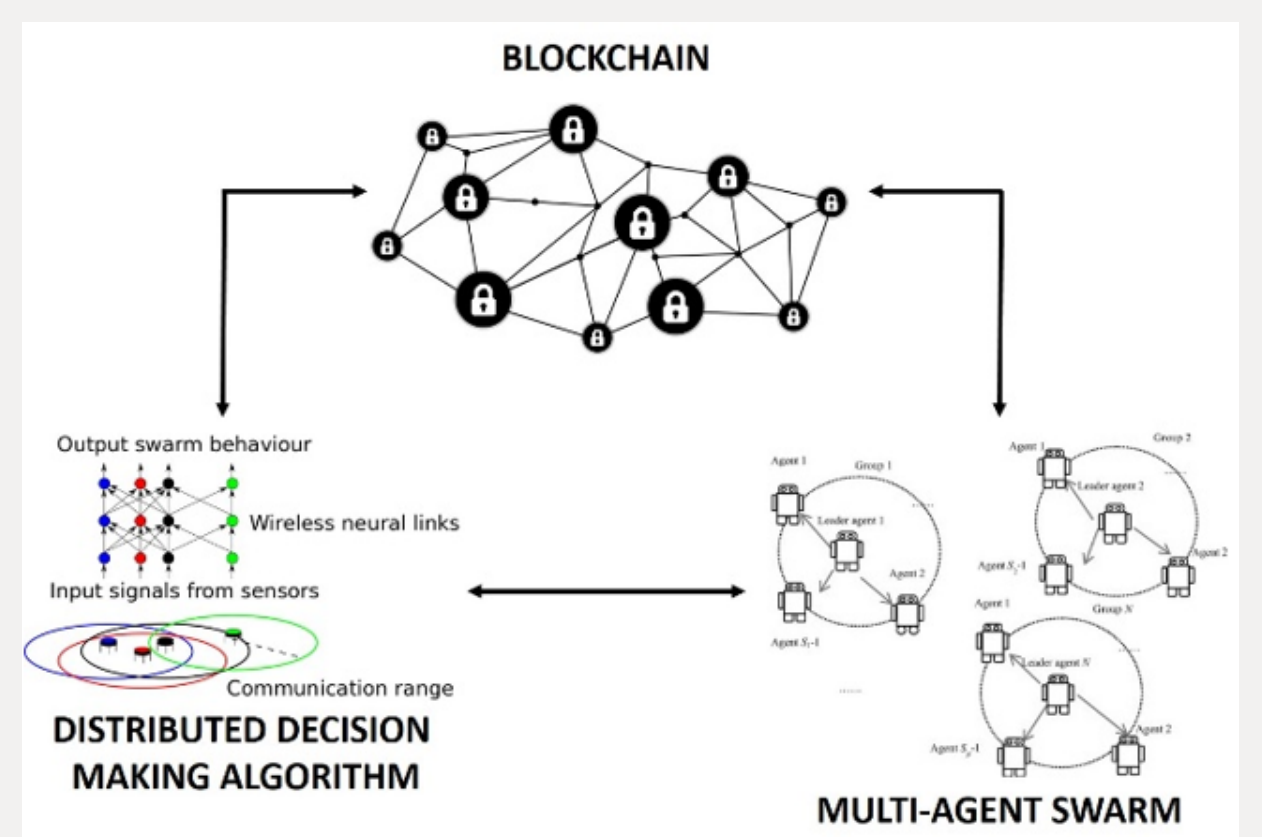

Figure 1: Blockchain enabled robot swarm distributed decision-making algorithm.

\section{Conclusion}

The latest advances in distributed decision-making algorithms provide swarm robotic systems with robot autonomy, decentralized control, and collective emergent behavior. The implementation of blockchain technology in distributed decision-making algorithms shows excellent potential for innovative solutions and progress in swarm robotic systems. This can open a possibility for robots to function in undetermined and changing environments without changing the control algorithms.

\section{References}

1. Mandrita Banerjee, Junghee Lee, Kim-Kwang Raymond Choo (2018) A blockchain future for internet of things security: a position paper. Digital Communications and Networks 4(3): 149-160.
2. Y Meng, Q Chen, A Rahmani (2018) A decentralized cooperative control scheme for a distributed space transportation system. Robotics and Autonomous Systems 101: 1-19.

3. Daniel Minoli, Benedict Occhiogrosso (2018) Blockchain mechanisms for IoT security. Internet of Things 1(2): 1-13.

4. NM Kumar (2018) Blockchain: Enabling wide range of services in distributed energy system. Beni-Suef University Journal of Basic and Applied Sciences.

5. Madhusudan Singh, Shiho Kim (2018) Branch Based Blockchain Technology in Intelligent Vehicle. Computer Networks 145: 219-231.

6. Teddy M Cheng, Andrey V Savkin, Faizan Javed (2011) Decentralized control of a group of mobile robots for deployment in sweep coverage. Robotics and Autonomous Systems 59(7-8): 497-507.

7. Lorenzo Sabattini, Cristian Secchi, Alessio Levratti, Cesare Fantuzzi (2015) Decentralized Control of Cooperative Robotic Systems for 
Arbitrary Setpoint Tracking while Avoiding Collisions. IFAC-Papers OnLine 48(19): 57-62.

8. E Ferrera, Jesus Capitan, Angel R Castano, Pedro J Marron (2017) Decentralized safe conflict resolution for multiple robots in dense scenarios. Robotics and Autonomous Systems 91: 179-193.

9. Levent Bayındır (2016) A review of swarm robotics tasks. Neurocomputing 172: 292-321.

10. Carlos Bentes and Osamu Saotome (2012) Dynamic Swarm Formation with Potential Fields and A* Path Planning in 3D Environment. 2012
Brazilian Robotics Symposium and Latin American Robotics Symposium (74-78).

11. Manuele Brambilla, Eliseo Ferrante, Mauro Birattari, Marco Dorigo (2013) Swarm robotics: a review from the swarm engineering perspective. Swarm Intelligence 7(1): 1-41.

12. S Pourmehr, VM Monajjemi, R Vaughan, and G Mori (2013) You two! take off!: Creating, modifying and commanding groups of robots using face engagement and indirect speech in voice commands. In Intelligent Robots and Systems (IROS).

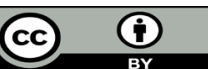

This work is licensed under Creative Commons Attribution 4.0 License

To Submit Your Article Click Here: $\quad$ Submit Article

DOI: $10.32474 /$ ARME.2018.01.000116

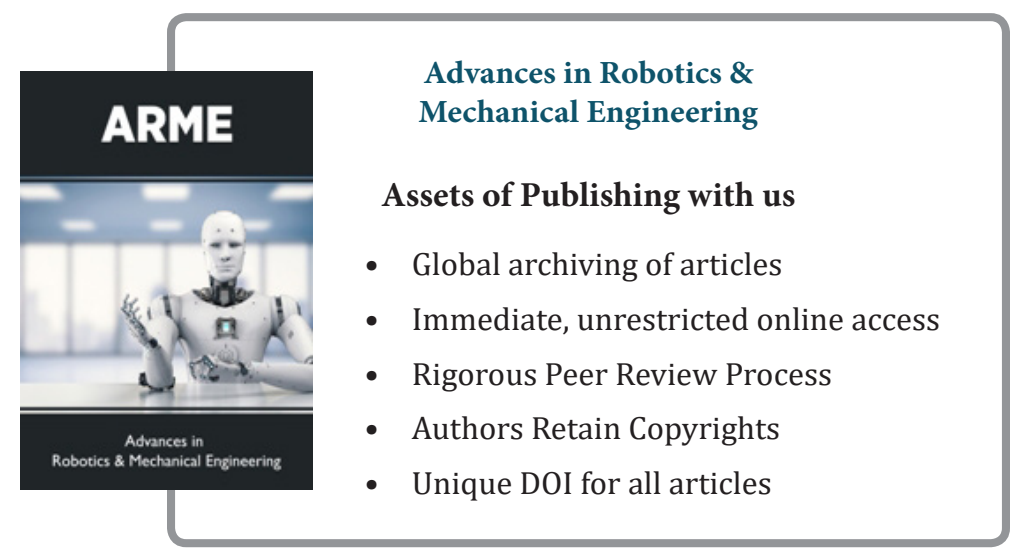

